\title{
Familial Hypocalciuric Hypercalcemia (FHH): an important differential diagnosis from hyperparathyroidism
}

I. Luque-Fernández ${ }^{1}$, A. Vicente, A. Luque-Pazos ${ }^{1}$,F. Del Val-Zaballos¹, I. Garin², MA. Morlan³. 1 Endocrinology Division, Hospital Virgen de la Salud, Toledo, Spain. 2 Molecular genetics laboratory, UH Araba-Txagorritxu, Vitoria, Spain. 3 Surgery Department, Hospital Virgen de la Salud, Toledo, Spain.

Introduction: Familial hypocalciuric hypercalcemia $(\mathrm{FHH})$ is a rare, benign, autosomal dominant condition usually caused by heterozygous inactivating mutations in the gene encoding the calcium sensing receptor (CASR).

Case: 22 year old woman who was referred to endocrinology for suspected polycystic ovary syndrome. Along de initial study hypercalcemia was detected and further laboratory tests showed persistent hypercalcemia with high iPTH levels.

Bioquemical data:

\begin{tabular}{|c|c|}
\hline Calcium (mg/dl) & $11.2(8.8-10.2)$ \\
\hline Phsophorus ( mg/dl) & $2.8(2.7-4.5)$ \\
\hline iPTH (pg/ml) & $101.7(10-65)$ \\
\hline $250 H v i t a m i n ~ D(m g / d l)$ & $17(2.7-4.5)$ \\
\hline
\end{tabular}

Urine calcium $\longrightarrow 186.9 \mathrm{mg} / 24 \mathrm{~h}$
Fractional excretion of calcium $\longrightarrow 0.18 \%$
Calcium/creatinine clearance ratio $(\mathrm{CCCR}) \longrightarrow 0.016$

Imaging: Parathyroid ultrasound and scintigraphy revealed no changes, and MRI showed and image of $4 \times 4 \mathrm{~mm}$ behind the left thyroid lobe consistent with parathyroid adenoma.

Treatment: Upon the diagnosis of Primary hyperparathyroidism (PHPT), upper left parathyroidectomy is performed after having identified the four glands. Histological analysis showed a normal parathyroid tissue.

Evolution: Persistent postsurgical high levels of calcium and iPTH prompt us to consider the diagnosis of FHH and to request a genetic study that revealed the presence, in heterozygosity, of the pathogenic mutation c.2089 G>A (p.Val697Met) in exon 7 of CaSR gene. Biochemical evaluation and genetic analysis was carried out on relatives, finding mild hypercalcemia, normal/high PTH and genetic mutation in her brother and father as well as in other paternal family members.

Conclusion: FHH is a rare but important cause of hypercalcemia, especially in the younger population because its presentation significantly overlaps with that of the much more common PHPT with respect to almost any clinical variable except the CASR gene test. The diagnosis is important in order to avoid unnecessary therapeutic interventions.

The CCCR can help differentiate but it has its limitations related to an indeterminate range between 0.01 and 0.02 where the diseases continue to overlap. Genetic testing for mutations in the CaSR gene is the only method for confident diagnosis of $\mathrm{FHH}$. 